\title{
The ubiquitin ligase mLin41 temporally promotes neural progenitor cell maintenance through FGF signaling
}

\author{
Jianfu Chen, ${ }^{1,3}$ Fan Lai, ${ }^{2}$ and Lee Niswander ${ }^{1,3}$ \\ ${ }^{1}$ Howard Hughes Medical Institute, Department of Pediatrics, University of Colorado Anschutz Medical Campus, Children's \\ Hospital Colorado, Aurora, Colorado 80045, USA; ${ }^{2}$ The Wistar Institute, Philadelphia, Pennsylvania 19104, USA
}

\begin{abstract}
How self-renewal versus differentiation of neural progenitor cells is temporally controlled during early development remains ill-defined. We show that mouse Lin41 ( $m L i n 41)$ is highly expressed in neural progenitor cells and its expression declines during neural differentiation. Loss of $m$ Lin41 function in mice causes reduced proliferation and premature differentiation of embryonic neural progenitor cells. mLin 41 was recently implicated as the E3 ubiquitin ligase that mediates degradation of Argonaute 2 (AGO2), a key effector of the microRNA pathway. However, our mechanistic studies of neural progenitor cells indicate mLin41 is not required for AGO2 ubiquitination or stability. Instead, mLin41-deficient neural progenitors exhibit hyposensitivity for fibroblast growth factor (FGF) signaling. We show that mLin41 promotes FGF signaling by directly binding to and enhancing the stability of She SH2-binding protein 1 (SHCBP1) and that SHCBP1 is an important component of FGF signaling in neural progenitor cells. Thus, $\mathrm{mLin} 41$ acts as a temporal regulator to promote neural progenitor cell maintenance, not via the regulation of AGO2 stability, but through FGF signaling.
\end{abstract}

[Keywords: mLin41; neural progenitor cells; AGO2; ubiquitination; FGF signaling; SHCBP1; neural tube closure defect] Supplemental material is available for this article.

Received January 18, 2012; revised version accepted March 5, 2012.

The mammalian CNS arises from a single layer of neural progenitor cells named neuroepithelial cells. During early neural development, neuroepithelial cells line the lumen of the ventricles and undergo rapid symmetric cell division to expand neural progenitor cell populations as the neural tube grows (Gotz and Huttner 2005). As development proceeds, neurogenesis is initiated as neural progenitor cells begin asymmetric cell divisions, exit the cell cycle, and migrate away from the lumen, giving rise to postmitotic cells that differentiate into distinct neurons, astrocytes, and oligodendrocytes. Distinct aspects of neurogenesis have been extensively studied, including cell cycle regulation (Ohnuma and Harris 2003), cell migration (Bielas et al. 2004; Ayala et al. 2007), and neural differentiation (Guillemot 2005). However, it remains undefined how neuroepithelial cells undergo rapid symmetric cell division and simultaneously maintain an undifferentiated state before the onset of neurogenesis in early development.

Fibroblast growth factor (FGF) signaling plays a crucial role in the regulation of neural progenitor cell proliferation

${ }^{3}$ Corresponding authors.

E-mail lee.niswander@ucdenver.edu.

E-mail jianfu.chen@ucdenver.edu.

Article is online at http://www.genesdev.org/cgi/doi/10.1101/gad.187641.112. and differentiation (Mason 2007). FGF signaling promotes proliferation of neural progenitor cells as well as represses premature neural differentiation (Kang et al. 2009), and the onset of neuronal differentiation requires the attenuation of FGF signaling (Diez del Corral et al. 2002). Despite the connection between FGF signaling and the maintenance of neural progenitor cells, it is ill-defined how the maintenance of neural progenitor cells is controlled in a spatiotemporal manner by FGF signaling and how that control is released and FGF signaling is attenuated to allow neural differentiation.

Mouse Lin41 (mLin41) is a member of the TRIM-NHL protein family and the target of let-7, a founding member of the microRNA (miRNA) family (Slack et al. 2000; Schulman et al. 2005; Kanamoto et al. 2006). Although recent studies in cultured cells suggest that mLin41 mediates Argonaute 2 (AGO2) degradation through an ubiquitination-dependent process (Rybak et al. 2009), whether mLin41 functions as an AGO2 regulator in vivo remains unknown. Mouse genetic data underscore the importance of mLin41 in embryonic development and neural tube closure (Maller Schulman et al. 2008). However, the cellular functions and underlying mechanisms of mLin41 action during development have not been studied. In the present study, we identify mLin41 as a temporal regulator of neural progenitor cell maintenance, and it acts by regulating 
FGF signaling. Mechanistically, mLin41 does not function as an E3 ubiquitin ligase to regulate AGO2 stability in neural progenitor cells. Instead, mLin41 interacts with and ubiquitinates She SH2-binding protein 1 (SHCBP1) and promotes SHCBP1 protein stability. Moreover, SHCBP1 regulates FGF signaling in neural progenitor cells. Together our data indicate that mLin41 and SHCBP1 provide temporal control of FGF signaling in neural progenitor cells.

\section{Results}

mLin41 expression declines during neuroepithelial cell differentiation

To identify the genes and mechanisms underlying neuroepithelial cell maintenance, we screened the developmental expression patterns of genes that, when disrupted, cause neural tube closure defects (data not shown). We reasoned that genes that specifically regulate neural progenitor cell function might exhibit decreased expression levels as neuroepithelial cells undergo differentiation. Among the genes examined, mLin 41 was highly expressed in neuroepithelial cells, and its expression declined as neurogenesis proceeded, as revealed by Western blot (Fig. 1A, complementary pattern to neural differentiation marker TuJ1) and in situ hybridization analyses (Fig. 1B,C). The temporal expression pattern of mLin 41 was confirmed by $\mathrm{X}$-gal staining of embryos from $m L i n 41^{1 a c Z /+}$ mice in which $\beta$-galactosidase expression is reflective of the endogenous mLin41 expression pattern (Fig. 1D-G). These results raise the possibility that mLin 41 is a temporal regulator of neural progenitor cell function.

\section{mLin41 is required for neural tube growth}

To assess mLin41 function in neural progenitor cells, we generated mLin41 mutant mice using gene trap embryonic stem (ES) cells in which the function of mLin 41 has been disrupted by the insertion of a $\beta$-geo reporter. mLin 41 is comprised of one ring finger domain, two B-box domains, one coiled-coil domain, one filamin, and six NHL domains (Fig. 2A). The insertion site was mapped to the intronic region flanked by exons 1 and 2 of mLin41. Western blot analyses confirmed the absence of mLin41 protein in homozygous mutant embryos (mLin41 lacZ/lacZ) (Fig. 2B).

mLin41 heterozygous mice were viable, fertile, and morphologically indistinguishable from their wild-type littermates. Analyses of litters derived from crosses of heterozygous mice showed no morphological difference between homozygous mutant and wild-type embryos at embryonic day 8.5 (E8.5) (data not shown). However, mutant embryos showed failure of cranial neural tube closure at E9.5 (also described in Maller Schulman et al. 2008) but otherwise were morphologically similar to their wild-type littermate. The Mendelian frequency of mLin41 homozygotes decreased by E10.5, and no viable mLin41 ${ }^{\text {lac } Z / l a c Z}$ embryos were recovered at E13.5 (Fig. 2C). In addition to the cranial neural tube defects (NTDs), there was also a pronounced growth retardation of the neural tissue in $100 \%$ of E10.5 mLin41 ${ }^{\text {lacZ/lacZ }}$ embryos (Fig. 2D-F). At E10.5, $92 \%$ of the $m L i n 41^{\text {lac } Z / l a c Z}$ mutants were slightly smaller $(80 \%-90 \%)$ than their somite-matched wild-type control littermates (Fig. 2E). A small percentage of E10.5 mutant embryos $(8 \%)$ had the expected number of somites, but the embryos were extremely small with NTDs (Fig. 2F). Together, these data show that mLin41 is required for proper neural tube growth and survival around mid-gestation.

\section{Loss of mLin41 reduces proliferation in neuroepithelial cells}

A role for mLin41 in neural tube closure was also reported by Maller Schulman et al. (2008), but the underlying cellular and mechanistic deficits were not defined. The failure of cranial neural tube closure and reduced size of the early neural tissue (Fig. 2D-F) suggested a defect of the neuroepithelial cells in mLin41 mutant embryos. This could be due to deficiency of neural progenitor cell proliferation (number and/or length of cell cycles), cell fate changes (fate specification or premature differentiation), defects in patterning or cell survival, or any combination of these factors. To determine whether mLin41 is involved
A
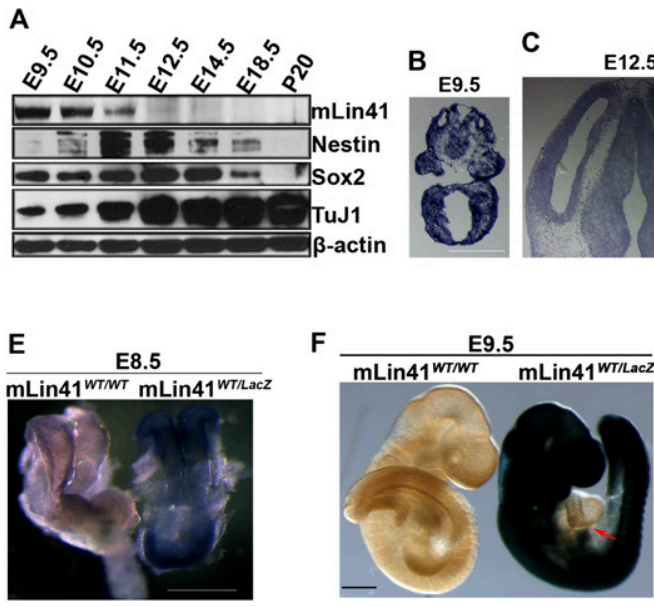
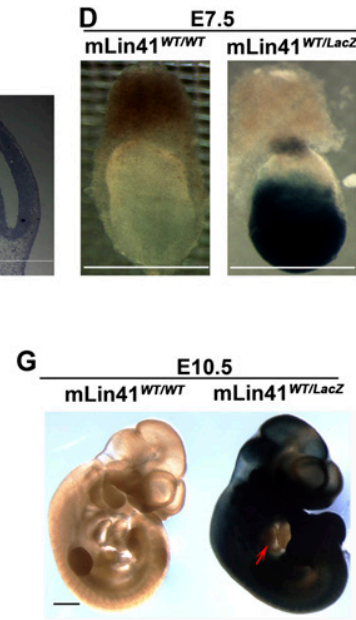

Figure 1. mLin41 expression declines during neural differentiation. (A) Western blot analyses of the protein expression of mLin 41 and neural markers in cranial neuroepithelium at different embryonic stages as indicated (represented by " $\mathrm{E}$ " for days post-coitum). $\beta$-Actin serves as a loading control. $(B, C)$ In situ hybridization analyses of mLin41 mRNA expression in sections of E9.5 and E12.5 embryos. Bars, $0.1 \mathrm{mM}$. $(D-G)$ Wholemount X-gal-stained embryos at E7.5 $(D)$, E8.5 $(E)$, E9.5 $(F)$, and E10.5 $(G)$. Red arrows indicate the absence of $m \operatorname{Lin} 41$ expression in the heart at E9.5 and E10.5. Bars, 0.5 mM. 

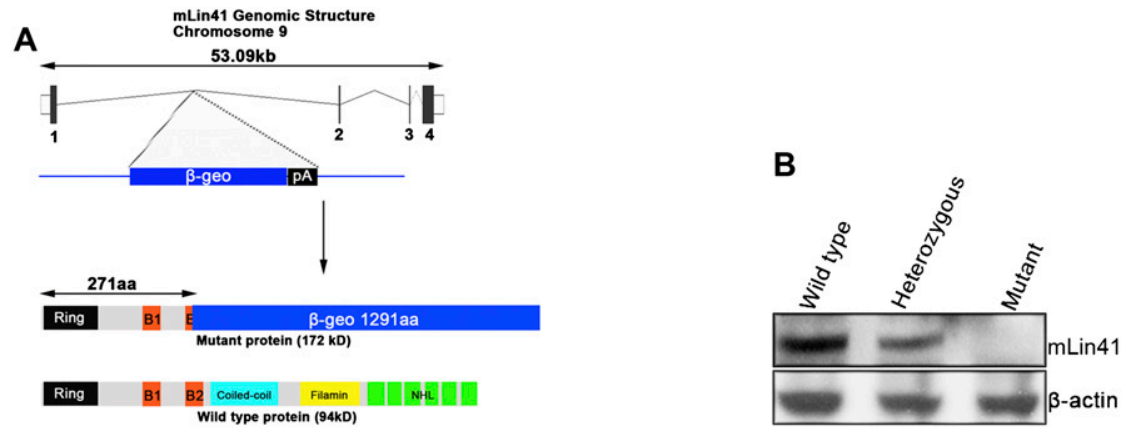

\begin{tabular}{|c|c|c|c|}
\hline Stage & WT/WT & $\mathrm{mLin} 41^{\text {WTAac } Z}$ & $\mathrm{mLin} 41^{\text {lacZ } I a c Z}$ \\
\hline E8.5 & 45 & 88 & 42 \\
\hline E9.5 & 51 & 103 & 54 \\
\hline E10.5 & 39 & 80 & 25 \\
\hline E13.5 & 44 & 86 & 0 \\
\hline
\end{tabular}
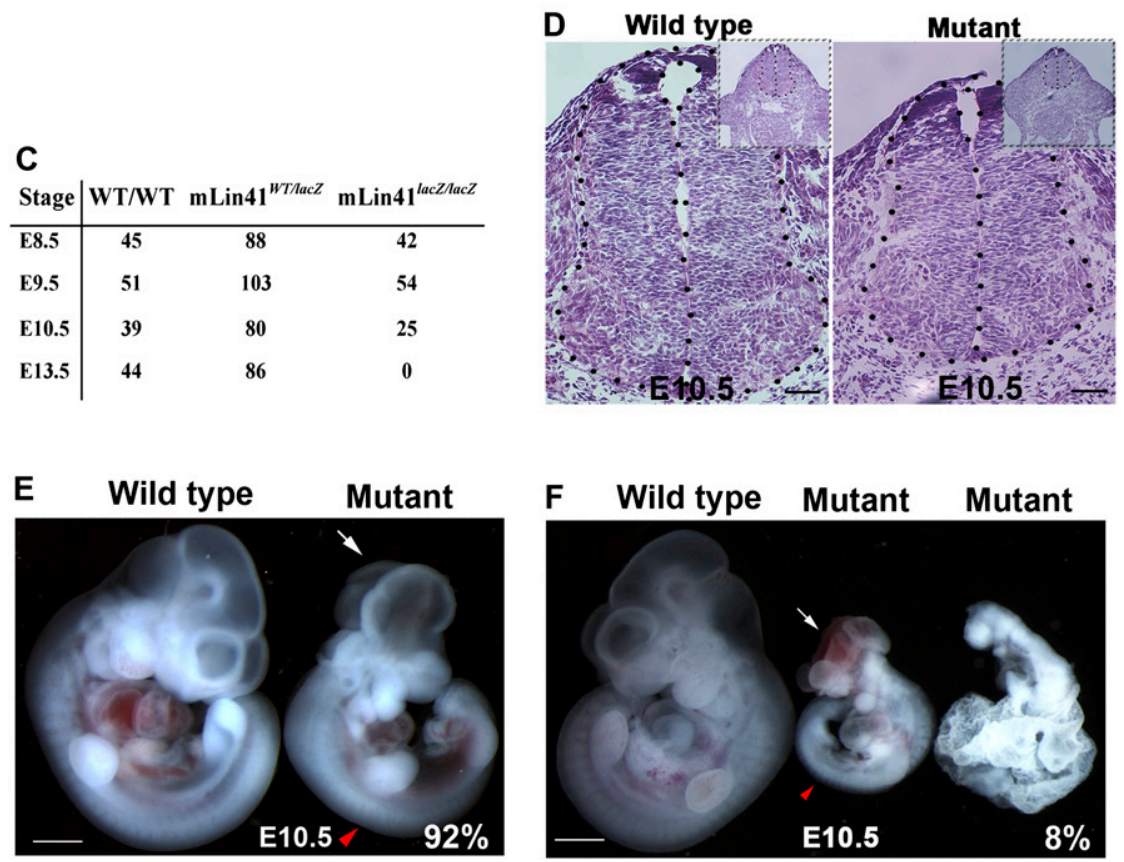

Figure 2. $\mathrm{mLin} 41$ is required for neural tube growth. (A) Genomic structure of mLin41 locus on mouse chromosome 9, and the gene trap vector pGT1lxf (BayGenomics) is inserted between exons 1 and 2. Wild-type mLin 41 protein is $\sim 94 \mathrm{kDa}$, whereas the mutant protein arising from the fusion between the mLin $41 \mathrm{~N}$ terminus (271 amino acids) and $\beta$-geo is $\sim 172 \mathrm{kDa}$. $(B)$ Western blot analyses of $\mathrm{mLin} 41$ protein expression in cranial neuroepithelium of E9.5 wild-type, heterozygous, and mLin41 1 lacz/lacz mutant embryos using an antibody that recognizes the $\mathrm{C}$ terminus of mLin41. $\beta$-Actin as loading control. $(C)$ Number of wild-type (WT/WT), heterozygous (mLin $41^{\text {WT/lacZ }}$ ), and mutant (mLin41 lacZ/lacZ) embryos recovered alive from litters dissected at the indicated embryonic stages. $(D) \mathrm{H} \& \mathrm{E}$ staining of transverse sections from E10.5 wild-type and mutant neural tubes at comparable rostral-caudal levels. Black dots outline neural tubes, and small insets indicate the whole sections. Bar, $35 \mu \mathrm{m}$. (E) Ninety-two percent of E10.5 mutant embryos exhibit open neural tube (white arrow) and reduced neural tissue growth (red arrowhead) compared with littermate control embryos. Bar, $0.5 \mathrm{mM}$. $(F)$ Eight percent of E10.5 mutants show dramatic growth retardation compared with littermate control embryos. Bar, $0.5 \mathrm{mM}$. in specification of neural progenitor cells, we examined the expression of Hes5, a neural progenitor specification marker (Ohtsuka et al. 1999). In situ hybridization analyses at E8.5 showed appropriate expression of Hes5 in mutant embryos, indicating that neural progenitor cell fate is properly specified in $\mathrm{mLin} 41^{\text {lacZ/lacZ }}$ mutants (Supplemental Fig. 1A).

Programmed cell death occurs during normal CNS development (Kuan et al. 2000), and the reduced neural tube size could be due to increased apoptotic cell death in mutant embryos. TUNEL staining of comparable coronal sections through wild-type and mutant neural tubes at E9.5 or E10.5 showed no significant increase in cell death in mutant embryos (Supplemental Fig. 1B-D), although by E11.5, there was increased cell death in the mutant hindbrain compared with wild type (Supplemental Fig. $1 \mathrm{E}, \mathrm{F})$. These studies suggest that the reduction in neuroepithelial cells in mLin41 mutants at the time of neural tube closure (E9.5) cannot be explained by increased cell death.

Bone morphogenetic protein (BMP) and Sonic hedgehog (SHH) signaling regulate transcriptional networks to pat- tern the neural tube along the dorsal-ventral (DV) axis (Jessell and Dodd 1990; Briscoe et al. 2000), and disruption of this process can lead to NTDs (Copp et al. 2003). To assess whether the NTDs in mLin41 lacZ/lacZ mutants result from DV patterning defects, we examined the expression of markers reflective of the neural progenitor positions along the DV axis of the neural tube. In E9.5 mutant embryos, the spatial distribution of neural progenitor markers resembled that in somite-matched control embryos: Msx1/2, Pax3, Pax6, Nkx2.2, and Shh for dorsal, intermediate, and ventral neural tube, respectively (Supplemental Fig. 2). Furthermore, Western blot analyses showed appropriate expression levels of phospho-Smad1/ $5 / 8$, reflective of BMP signaling in mutant neuroepithelium (p-Smad1,5,8 expression in Fig. 6A,B). Together, these results suggest that DV patterning is not disrupted in mLin41 mutant neural tubes and the neural tube closure defects in mLin41 mutants are not due to DV patterning defects.

To examine whether the reduction in neuroepithelium in mutant embryos resulted from decreased mitotic rates, we used phospho-histone $\mathrm{H} 3$ (p-H3) antibody to label 
Chen et al.

mitotic cells. In E9.5 wild-type embryos, p-H3-positive neural progenitor cells form a nearly continuous outline around the ventricular surface of the neuroepithelium (Fig. 3A). mLin41 mutant embryos exhibit a reduction of p-H3-positive cells (Fig. 3A), confirmed by quantitation of p-H3-positive cells in the neuroepithelium $(n=6$ wildtype and mutant embryos) (Fig. 3B) and Western blot analyses ( $n=3$ wild-type and mutant embryos) (Fig. 3C). Interestingly, some p-H3-positive cells appear on the lateral sides of the mutant neuroepithelium (Fig. 3A, white arrowheads), which is rarely observed in wild type, indicating that some p-H3-positive cells are mislocalized in the mutants. Since p-H3 only labels cells in $M$ phase, we examined cells in $\mathrm{S}$ phase by performing bromodeoxyuri-
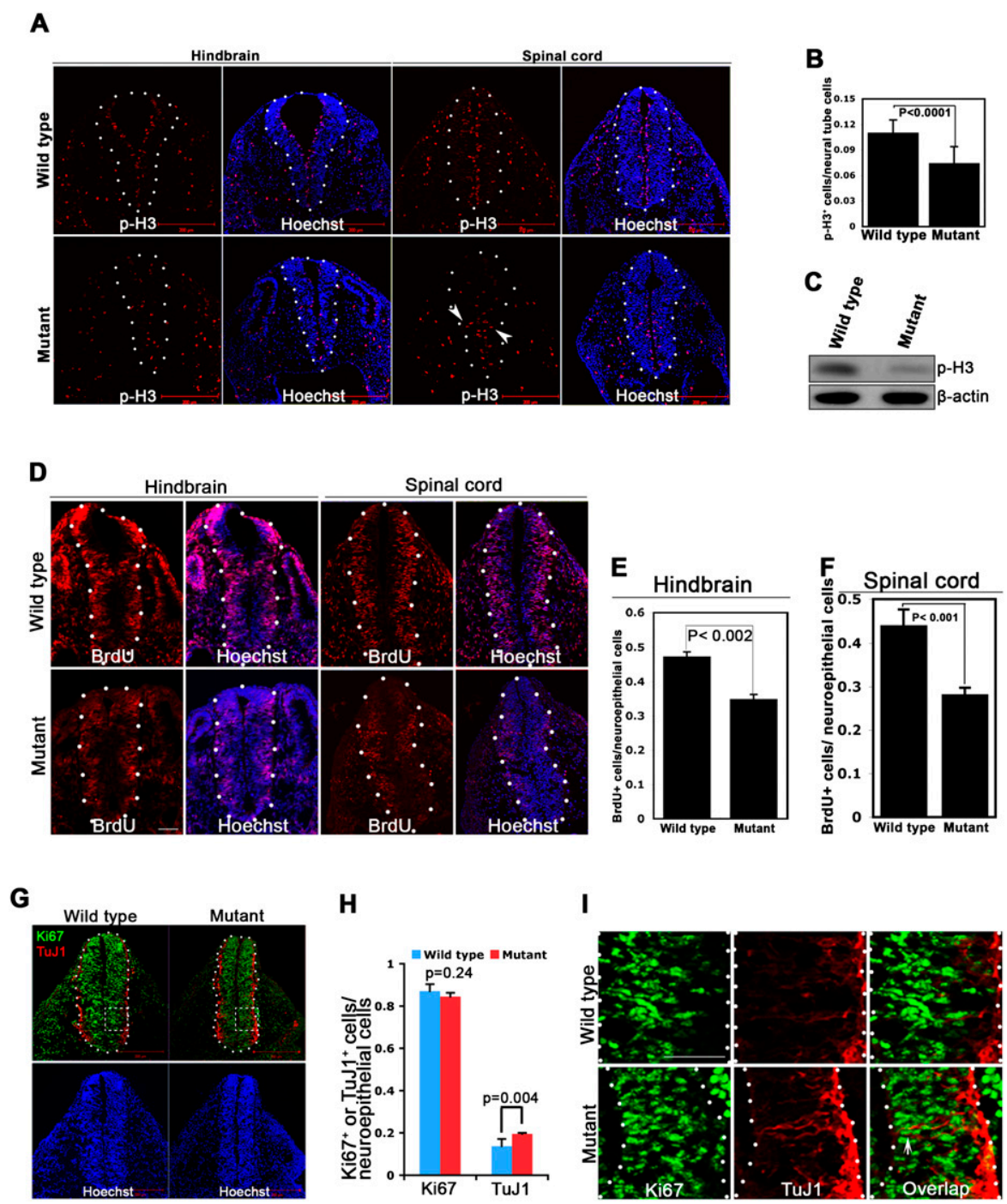

Figure 3. mLin41 deficiency leads to reduced cell proliferation in the neuroepithelium. $(A)$ Confocal microscope images of sections from hindbrains and spinal cords of E9.5 wild-type and $m L i n 41^{\text {lacZ/lacZ }}$ mutant embryos. Anti-p-H3 antibody visualizes mitotic cells (red). White arrowheads indicate mislocalized p-H3-positive cells in the mutant neuroepithelium. Hoechst stains nuclei (blue). Bar, 200 $\mu \mathrm{m}$. (B) Quantitation of p-H3-positive cells from A. Percentage of p-H3-positive cells was calculated as the percentage of p-H3-positive cells out of the total number of neuroepithelial cells, indicated by Hoechst staining, within the neural tube sections. Error bars indicate SEM of 12 sections from three independent experiments. $P<0.0001 .(C)$ Western blot analyses of the expression level of p-H3 in E9.5 wild-type and mutant cranial neuroepithelium. $\beta$-Actin serves as a loading control. $(D)$ Confocal microscope images of hindbrain and spinal cord sections from E9.5 wild-type and mutant embryos after $1 \mathrm{~h}$ of BrdU labeling. S-phase cells were visualized by anti-BrdU antibody (red); Hoechst stains nuclei (blue). Bar, $100 \mu \mathrm{m} .(E, F)$ Quantitation of percentage of BrdU-positive cells per total number of neuroepithelial cells indicated by Hoechst staining in hindbrain and spinal cord sections. Error bars indicate SEM of 12 sections from three independent experiments. $(G, I)$ Confocal microscope images of spinal cord sections from E9.5 wild-type and mutant embryos immunostained with anti-Ki67 antibody (green) to label cycling cells and anti-TuJ1 antibody (red) to label differentiated neurons. $I$ is high magnification of the areas outlined by white boxes in $G$. White dots outline the neuroepithelial cells, and the white arrowhead indicates cells that coexpress Ki67 and TuJ1 in mutants. Bars: $G, 200 \mu \mathrm{m} ; I, 50 \mu \mathrm{m}$. $(H)$ Quantitative measurement of Ki67-positive cells or TuJ1-positive cells counted from each neural tube section of wild-type and mutant embryos. Error bars indicate SEM of nine sections from three independent experiments. 
dine (BrdU)-labeling experiments. Loss of mLin41 resulted in a significant reduction of BrdU-positive cells in mutant compared with wild-type neuroepithelium $(n=5$ wild-type and mutant embryos) (Fig. 3D-F). Together, these two markers of the cell cycle indicate reduced proliferation of neural progenitor cells in the mutant embryos.

To ask whether mutant neural progenitor cells prematurely exit the cell cycle, undergo cell cycle arrest, or differentiate, we used Ki67 to mark cells in all phases of the cell cycle except G0 and TuJ1 to label differentiated neurons. Mutant neural progenitor cells do not prematurely exit the cell cycle, as revealed by similar spatial distribution of Ki67 in the E9.5 mutant neural tubes compared with wild type (Fig. 3G-I). However, some mutant cells expressed both Ki67 and TuJ1 markers (white arrowheads in Fig. 3I), whereas in wild type, these markers are mutually exclusive, suggesting premature differentiation of mutant neural progenitor cells. Thus, these data indicate that the decreased proliferation rate but not premature cell cycle exit of neural progenitor cells causes reduced neural tube growth in mLin41 mutants.

\section{Loss of mLin41 leads to premature differentiation of neural progenitor cells}

To examine neural differentiation in the mLin 41 mutant neuroepithelium, somite-matched E9.5 wild-type and mutant embryos at comparable anterior-posterior levels within the hindbrain and spinal cord were analyzed. First, we examined the expression of markers that define neural progenitors (Sox2/Nestin) or neurons that have started to differentiate (Neurofilament/TuJ1). In both the hindbrain and spinal cord, there was a slight down-regulation of neural progenitor markers Sox 2 and Nestin in mutants ( $n=5$ wild-type and mutant embryos) (Fig. 4A,B). In mutants, there was also a significant increase in cells expressing Neurofilament and TuJ1, indicating premature differentiation of neural progenitor cells $(n=5$ wildtype and mutant embryos) (Fig. 4A,B). A number of transcription factors function as intrinsic regulators of neural differentiation. Proneural genes such as Mash1, Math1, and Neurogenin are key regulators of neural differentiation, whereas Notch signaling negatively regulates neurogenesis by activating the Hairy enhancer of Split (HES) family of basic helix-loop-helix (bHLH) genes, Hes-1 and Hes-5 (Bertrand et al. 2002; Yoon and Gaiano 2005). We compared Notch1, Hes5, Mash1, Math1, and NeuroD expression between wild-type and mutant neuroepithelium. In the E9.5 neural tube, NeuroD and Math1 were barely detected in both wild-type and mutant embryos (data not shown), consistent with their later roles in neural differentiation (Bertrand et al. 2002). However, the expression of Notch1 and Hes5, repressors of neural differentiation, was significantly reduced in mutants compared with wild type ( $n=3$ wild-type and mutant embryos; Fig. 4C). Correspondingly, the proneural gene Mash1 was upregulated in mutants compared with wild type ( $n=3$ each) (Fig. 4C). Together, these data indicate that mLin41 maintains a neural progenitor state at least in part by repressing neural differentiation.
The reduced proliferation of neural progenitor cells and increased number and premature differentiation of neurons in mLin41 $1^{\text {lacZ/lacZ }}$ mutants led us to hypothesize that the prematurely differentiated cells were generated at the expense of neural precursor cells in the mutant neuroepithelium. To test this hypothesis, we examined the generation of motor neurons, which differentiate early in the ventral spinal cord (Jessell 2000). Olig2 and Is11/2 expression mark motor neuron progenitors and motor neurons, respectively (Pfaff et al. 1996; Novitch et al. 2001). In E9.5 mutant spinal cords, the number of Olig2positive progenitor cells was significantly reduced, and correspondingly, Isl1/2-positive motor neurons were increased ( $n=3$ wild-type and mutant embryos) (Fig. 4D-F), and this was confirmed by Western blot analyses $(n=3$ each) (Fig. $4 \mathrm{G}, \mathrm{H}$ ). This is consistent with the idea that premature differentiation of motor neurons comes at the expense of motor neuron progenitors in the $\mathrm{mLin} 41$ mutant neural tubes. Moreover, in E9.5 wild-type spinal cords, there was a mutually exclusive distribution pattern of Olig2 and Isl1/2 expression. However in mutant spinal cords, Olig2 and Isl1/2 double-positive cells were detected (white arrows in Fig. 4I), again indicating that motor neuron precursors prematurely differentiate in mLin 41 mutants. Taken together, these results support the model that mLin 41 is required to maintain proliferation and prevent premature differentiation of neural progenitor cells. Extending this logic, during normal neurogenesis, mLin41 is downregulated as neural differentiation proceeds, suggesting that mLin41 functions as a temporal switch between neural progenitor maintenance and differentiation.

mLin41 is dispensable for the regulation of AGO2 ubiquitination and stability in vitro and in vivo

To explore the underlying mechanisms of mLin41 function in the neuroepithelium, we first examined mLin41 localization in the NE-4C cell line established from the cerebral vesicle of E9.0 mouse embryos. NE-4C cells mimic neural progenitor cell proliferation and differentiation in vitro and express mLin41 (Supplemental Fig. 3A; Varga et al. 2008). Localization studies following transfection of various mLin41 constructs (Supplemental Fig. 3B) showed that mLin41 is localized to the cytoplasm, which requires the coiled-coil domain (Supplemental Fig. 3C). Previous studies showed that mLin41 is associated with AGO2 in the embryonic carcinoma cells (Rybak et al. 2009). We confirmed the physical interaction between mLin41 and AGO2 in NE-4C cells (Supplemental Fig. $3 \mathrm{D}, \mathrm{E})$. Using various tagged $\mathrm{mLin} 41$ constructs, we mapped the regions of mLin41 responsible for AGO2 binding (Supplemental Fig. 3F). Together, these findings suggest that the coiled-coil domain of mLin 41 is essential for its cytoplasmic localization and the association between mLin41 and AGO2.

Earlier studies in embryonal carcinoma cells implicated mLin41 as the E3 ubiquitin ligase that regulates AGO2 stability (Rybak et al. 2009). To test whether mLin41 promotes neural progenitor cell maintenance through the regulation of AGO2 turnover, we first confirmed that 


\section{A \\ A}

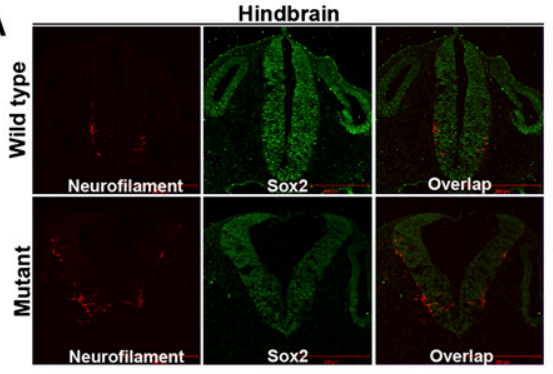

B

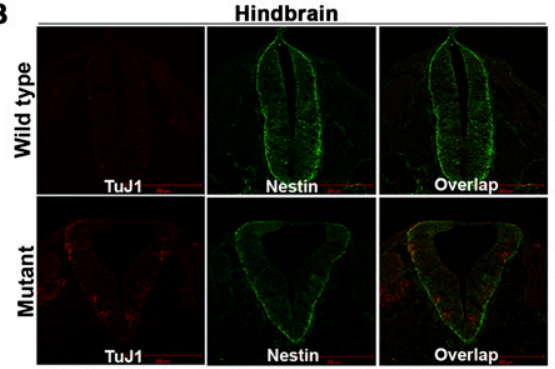

C
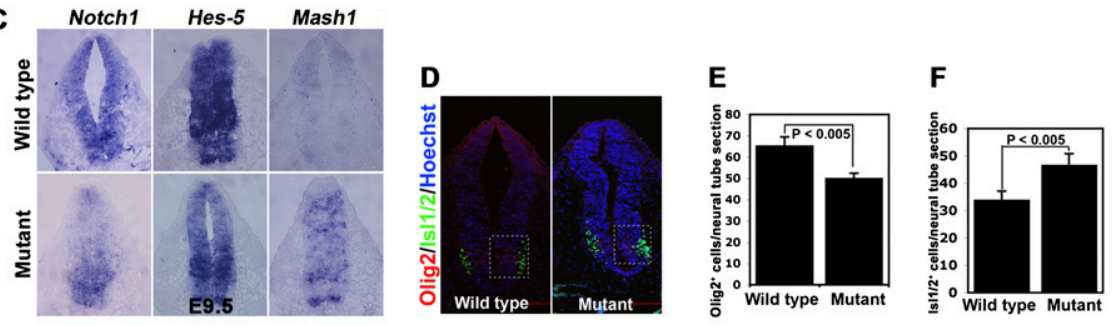

G

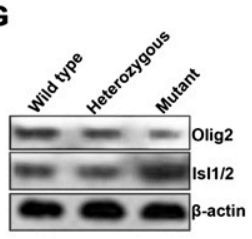

H

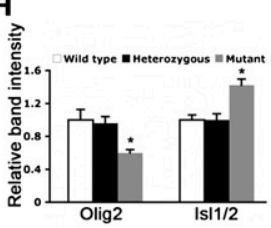

I

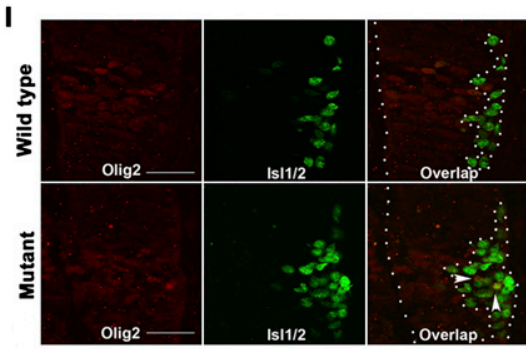

Spinal cord

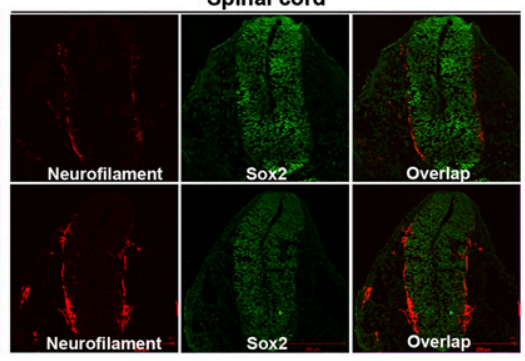

Spinal cord

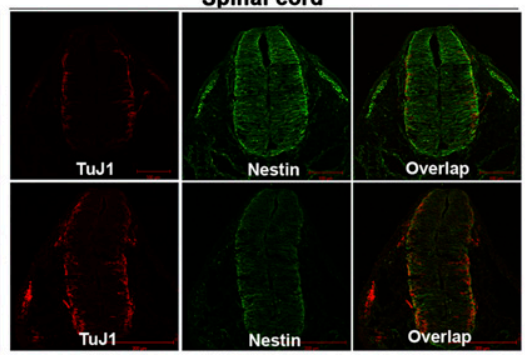

mLin41 is an E3 ubiquitin ligase with autoubiquitination activity, and this requires the RING finger domain (Fig. 5A; Rybak et al. 2009). However, Western blot analyses of transfected $293 \mathrm{~T}$ cells revealed that AGO2 protein levels are not significantly changed upon overexpression of mLin41 (Fig. 5B). Moreover, inhibition of the proteasome with MG132 showed that AGO2 protein levels are not increased (Fig. 5B), suggesting that AGO2 is not degraded by mLin41 in a proteasome-dependent manner. Next we examined cell lysates prepared from cranial neuroepithelium of E9.5 wild-type and mLin41 $1^{\text {lacZ/lacZ }}$ mutant embryos by Western blot and found that AGO2 protein levels are comparable in cells with or without mLin41 (Fig. 5C,D), indicating that deletion of mLin41 had no effects on the stability of AGO2 in neural progenitor cells. Similarly, there was no significant difference in the expression of other miRNA pathway components, including
Figure 4. Loss of mLin41 results in premature differentiation of neural progenitor cells. $(A, B)$ Confocal microscope images of sections from hindbrains and spinal cords of E9.5 wild-type and mLin41 1 lacZ/lacZ mutant embryos. Bar, $200 \mu \mathrm{m}$. (A) Anti-Neurofilament antibody (red) marks differentiated neurons, and anti-Sox2 antibody (green) labels neural progenitor cells. (B) AntiTuJ1 antibody (red) marks differentiated neurons, and anti-Nestin antibody (green) labels neural progenitor cells. Note that there are significantly more Neurofilamentand TuJ1-positive cells in the mutant neuroepithelium compared with littermate controls. $(C)$ In situ hybridization on spinal cord sections of E9.5 wild-type and mutant embryos with probes for neural progenitor markers Notch1 and Hes-5 and differentiation marker Mash1. $(D, I)$ Confocal microscope images of spinal cord sections from E9.5 wild-type and mutant embryos. Anti-Olig2 antibody (red) labels motor neuron precursors, and anti-Isl1/2 antibody (green) marks motor neurons. $I$ is high magnification of the areas outlined by white boxes in $D$. White dots in $I$ outline the neuroepithelial cells, and white arrowheads indicate Olig2- and Is11/2- double-positive cells in mutant neuroepithelium. Bars: $D$, $100 \mu \mathrm{m} ; I, 70 \mu \mathrm{m}$. $(E, F)$ Quantitative measurement of Olig2-positive cells $(G$; $P<0.005)$ and Isl1/2-positive cells $(H$; $P<0.005$ ) counted from each neural tube section of wild-type and mutant embryos. Error bars indicate SEM of 12 sections from three independent experiments. $(G)$ Western blot analyses of Olig2 and Isl1/2 expression in cranial neuroepithelium of E9.5 wild-type, heterozygous, and mutant embryos. $\beta$-Actin serves as a loading control. (H) Quantification of Western blot data using three independent blots.
DICER and AGO1, between wild-type and mLin41 mutant neuroepithelium (Fig. 5C,D). Theses studies suggest that mLin41 does not mediate AGO2 degradation in a proteasome-dependent manner in neural progenitor cells.

In addition to tagging proteins for degradation, ubiquitination is recognized as one of the mechanisms that regulate protein stability, protein-protein interaction, and cell signaling (Kirkin and Dikic 2007; Sorkin and von Zastrow 2009). To examine whether mLin41 ubiquitinates AGO2 without causing its degradation, we performed in vitro ubiquitination on AGO2, but these studies suggest that mLin41 does not enhance AGO2 ubiquitination (Supplemental Fig. 4A). Furthermore, we performed ubiquitination assays in NE-4C cells and HEK293T cells. The level of AGO2 ubiquitination is not increased upon overexpression of mLin41, and AGO2 ubiquitination is comparable between overexpression of wild-type mLin 41 
A

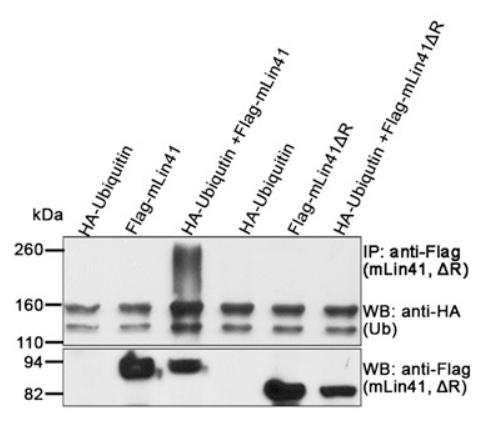

B

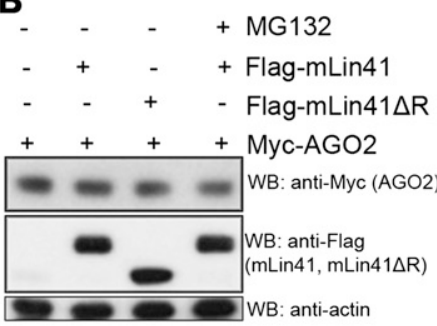

C

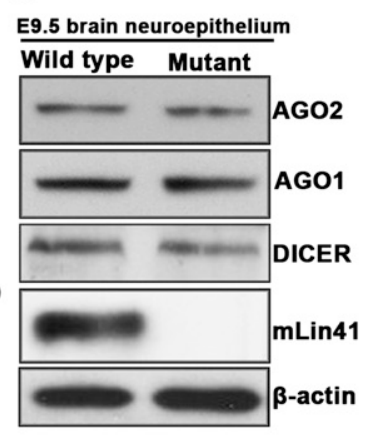

D

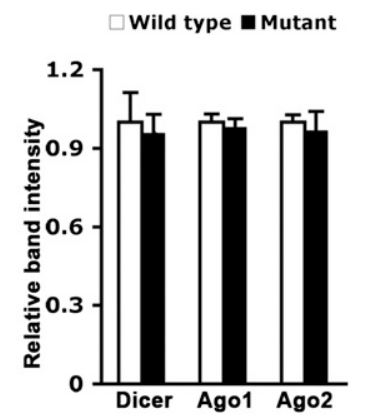

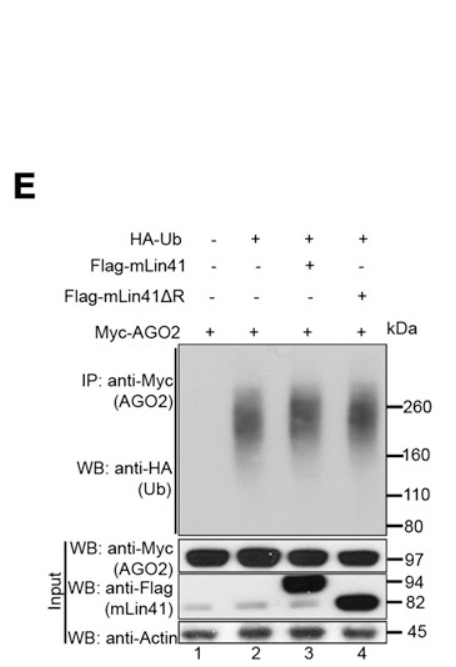
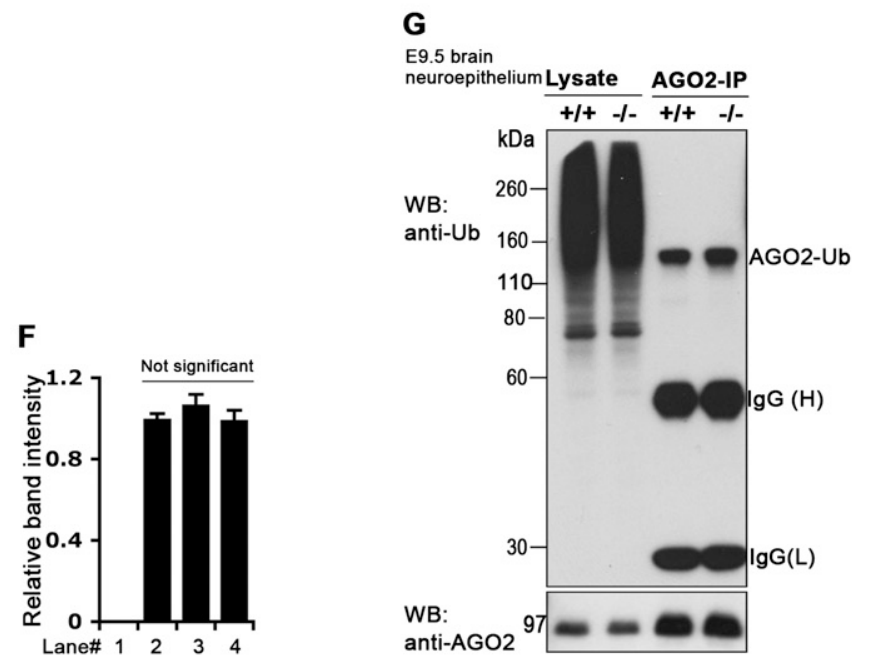

H

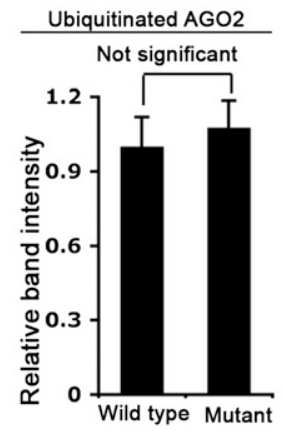

Figure 5. mLin 41 is dispensable for the regulation of AGO2 ubiquitination and stability in vitro and in vivo. $(A) \mathrm{mLin} 41$ is a RING finger-dependent ubiquitin ligase and is self-ubiquitinated. HA-tagged ubiquitin construct, Flag-tagged mLin41 construct (full-length or RING finger domain-depleted $[\Delta \mathrm{R}])$, or both constructs as indicated were transfected into 293T cells. Protein extracts were immunoprecipitated using anti-Flag beads (IP: $\alpha$-Flag) followed by Western blotting with anti-HA antibodies (WB: $\alpha$-HA). (B) mLin41 does not mediate AGO2 degradation in proteasome inhibition assays. 293T cells were transfected with plasmids as indicated. Twentyfour hours post-transfection, cells from the fourth lane were treated with the proteasome inhibitor MG132 (10 $\mu \mathrm{M})$ for $12 \mathrm{~h}$ before the Western blot analyses. AGO2 levels were not affected by the expression of mLin41 or the presence of MG132. (C) Western blot analyses of the expression of AGO2 and other miRNA pathway components as indicated in the cranial neuroepithelium of E9.5 wild-type and $m L i n 41^{1 a c Z / l a c Z}$ mutant embryos. $\beta$-Actin serves as a loading control. $(D)$ Quantification of Western blot data using three independent blots from $C$. $(E)$ In vivo ubiquitination assay of AGO2. Flag-tagged wild-type or RING finger domain-deleted mutants ( $\Delta$ R) of mLin41 were expressed in $293 \mathrm{~T}$ cells along with Myc-AGO2 and HA-ubiquitin (Ub) as indicated. The levels of AGO2 ubiquitylation were evaluated by the immunoprecipitation of AGO2 using anti-Myc antibodies followed by anti-HA immunoblotting. $(F)$ Quantification of Western blot data using three independent blots from E. (G) Endogenous AGO2 was immunoprecipitated from the cranial neuroepithelium of E9.5 wild-type and mLin41 ${ }^{\text {lacz/lacZ }}$ mutant embryos, and immunoprecipitates were immunoblotted with anti-ubiquitin antibody to detect ubiquitinated AGO2 (top blot) or anti-AGO2 antibody (bottom blot). The left two lanes represent the total ubiquitination of the lysate. $(H)$ Quantification of Western blot data using three independent blots from $G$.

or RING finger domain-deleted mutant mLin41 (Fig. 5E,F; Supplemental Fig. 4B). Next, we examined endogenous AGO2 ubiquitination levels in E9.5 mLin41 wild-type and mutant neuroepithelium. Experimental results show that the pattern and intensity of bands recognized by antiubiquitin antibodies (most likely representing ubiquitin-modified endogenous AGO2) were comparable in cells with or without mLin41 (Fig. 5G,H; Supplemental Fig. 4C,D). Together, our in vitro and in vivo results suggest that mLin41 does not directly mediate AGO2 ubiquitination or degradation in neural progenitor cells under phys- iological conditions. Therefore, our studies do not support the hypothesis that mLin41 promotes neural progenitor cell maintenance through the regulation of AGO2 ubiquitination or stability.

\section{mLin41-deficient neuroepithelial cells are hyposensitive to FGF signaling}

mLin41 is an E3 ubiquitin ligase that has autoubiquitination activities (Fig. 5A), and ubiquitination has emerged as a mechanism in the control of cell signaling (Kirkin and 
Dikic 2007; Sorkin and von Zastrow 2009). We hypothesized that mLin41 regulates neural progenitor cell maintenance through modulating cell signaling. Our analyses of neural tube patterning indicated that BMP and $\mathrm{SHH}$ signaling are not compromised in mLin41 mutants (Supplemental Fig. 2). FGF signaling plays important roles in maintaining neural progenitor cell self-renewal and proliferation (Mason 2007), and FGFR1 deficiency leads to NTDs (Deng et al. 1997). Therefore, we examined mLin41 mutants for changes in FGF signaling. MAPK/ERK1/2 and $\mathrm{PI}(3) \mathrm{K} / \mathrm{AKT}$ are downstream targets of FGF signaling and phosphorylation of ERK1/2, and AKT (p-ERK1/2 [Thr 202/ Thr 204] and p-AKT [Ser 473]) can be used as readouts of FGF signaling. Using extracts from E9.5 wild-type, heterozygous, and mutant cranial neuroepithelium, we observed that total levels of ERK1/2 and AKT were unaffected but endogenous activity, as indicated by phospho-ERK1/2 and phospho-AKT expression, was reduced in mLin 41 mutants (Fig. 6A,B). Immunostaining studies further confirmed the down-regulation of p-AKT in the mLin41 mutant compared with wild type (Fig. 6C). Consistent with down- regulation of AKT activity, the downstream substrate of AKT, GSK3 $\beta$, as revealed by anti-phospho-GSK3 $\beta$ (Ser9) antibody, was also significantly decreased, although total levels of GSK3 $\beta$ in mutants were normal (Fig. 6A,B). To determine whether the reduction of growth signaling in mLin 41 mutant neuroepithelium was due to the premature differentiation of neural progenitor cells, we performed Western blot analyses of p-AKT, p-ERK $1 / 2$, and p-GSK-3 $\beta$ in wild-type cranial neuroepithelium at different developmental stages but detected no significant change as the cells differentiate (Supplemental Fig. 5). Therefore, these results suggest that FGF signaling is defective in mLin 41 mutants. To explore this further, we examined the response of neuroepithelium to FGF2 stimulation. As shown by Western blot analyses (Fig. 6D-F), FGF2 treatment dramatically increased p-ERK1/2 and p-AKT levels in wildtype neuroepithelium. In comparison, $\mathrm{p}$-ERK1/2 and p-AKT levels were induced in mLin41 mutants but significantly less so than in wild-type embryos. Together, these results indicate that mLin41 regulates FGF signaling within neural progenitor cells and suggest that the decreased prolifer-
A

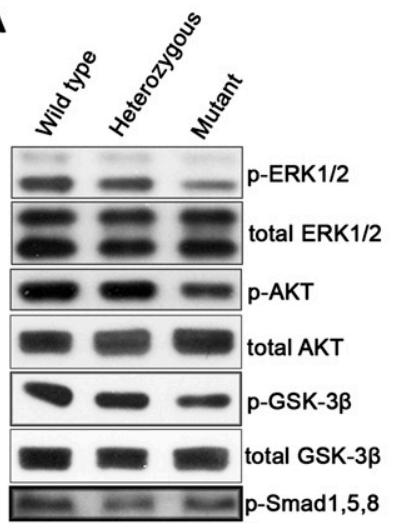

D

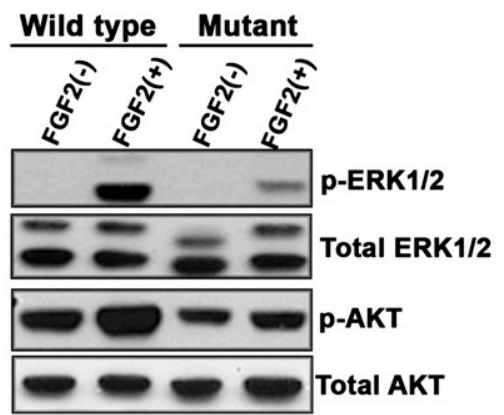

B

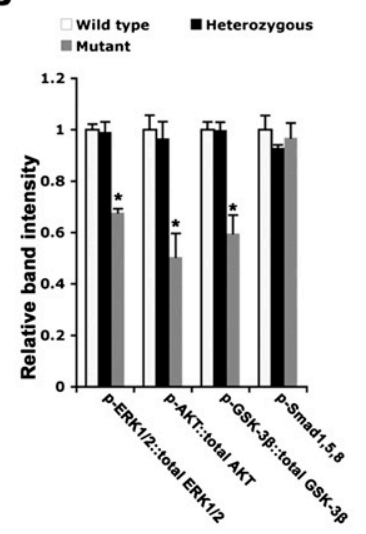

E

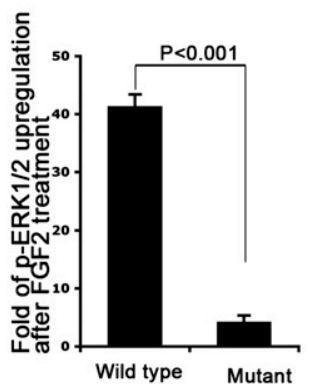

C

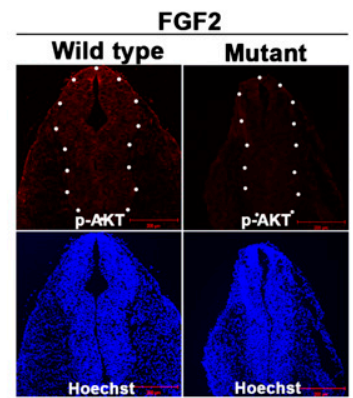

$\mathbf{F}$

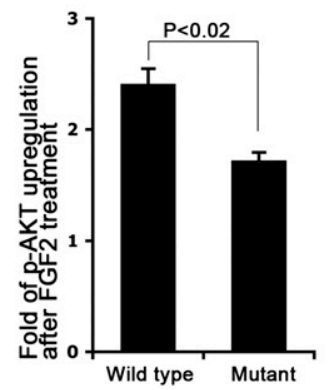

Figure 6. FGF signaling is defective in mLin41 mutant neuroepithelium. (A) Western blot analyses of the expression of indicated proteins in the cranial neuroepithelium of $\mathrm{E} 9.5$ wild-type, heterozygous $\mathrm{mLin} 41^{\text {lacZ/+ }}$, and $\mathrm{mLin} 41^{\text {lacZ/lacZ }}$ mutant embryos. $\beta$-Actin serves as a loading control. (B) Quantification of Western blot data from three independent blots in $A_{;}\left({ }^{\star}\right) P<0.005$. (C) Immunostaining of p-AKT (red) in spinal cord sections from E9.5 wild-type and mutant embryos grown in culture for 10 min with FGF2. Hoechst stains nuclei (blue). Bar, $200 \mu \mathrm{m}$. Note that fluorescence intensity of p-AKT is stronger in response to FGF2 in wild-type compared with mutant embryos. $(D)$ Western blot analyses of the expression of p-ERK1/2 and p-AKT in cranial neuroepithelium of E9.5 wild-type and mutant embryos grown in culture in the presence or absence or FGF2 for 10 min. Total ERK1/2 and total AKT expression serve as controls. Note the induction of p-ERK1/2 and p-AKT expression in response to FGF2 is strong in wild-type but is significantly less in mutant embryos. $(E, F)$ Quantification of Western blot data using three independent blots in $D$. 
ation and premature differentiation of neural progenitors in the mLin41 mutant is at least in part due to attenuated FGF signaling.

mLin41 enhances the stability of SHCBP1, an important component of FGF signaling in neural progenitor cells

To understand further how mLin41 is involved in the regulation of FGF signaling, we performed a yeast twohybrid screen and found that mLin41 interacts with SHCBP1 (Fig. 7A). SHCBP1 was identified by its interaction with SHC, which is involved in FGF signaling (Schmandt et al. 1999). Immunoprecipitation studies confirmed the physical interaction of mLin41/SHCBP1 (Fig. 7B). Given that mLin 41 is an E3 ubiquitin ligase (Fig. 5A), our further studies show that mLin41 significantly promotes SHCBP1 ubiquitination and deletion of the RING finger domain $(\mathrm{mLin} 41 \Delta \mathrm{R})$ significantly reduces the ubiquitination levels of SHCBP1 (Fig. 7C). Modification by ubiquitin can tag proteins for degradation, promote protein stability, or regulate protein-protein interactions (Kirkin and Dikic 2007; Sorkin and von Zastrow 2009). Therefore, we next examined the expression of different FGF signaling components, including SHCBP1, in mLin41 knockout embryonic neuroepithelium. This showed that the SHCBP1 protein level is significantly decreased in the mLin 41 mutant neuroepithelium, but FGFR1, SHC, and Sprouty2 (Spry2) levels are comparable with wild type (Fig. 7D,E). These studies suggest that mLin 41 positively regulates SHCBP1 protein expression in neural progenitor cells.

To test whether the E3 ubiquitin ligase activity of mLin41 is required for the regulation of SHCBP1 protein expression, we examined the effects of overexpression of mLin41 with or without the RING finger domain on SHCBP1 protein levels in HEK293T cells. Consistent with the in vivo data that mLin41 knockout decreases SHCBP1 protein levels (Fig. 7D,E), ectopic expression of mLin41 promotes SHCBP1 protein expression, and depletion of the RING finger domain blunts the degree of promotion (Fig. 7F,G). To test the possibility that mLin41 promotes SHCBP1 protein levels by affecting its protein stability, we used cycloheximide (CHX) to block protein synthesis and examined SHCBP1 degradation. SHCBP1 protein is greatly reduced within $10 \mathrm{~h}$, but mLin 41 significantly promotes the stability of SHCBP1 (Fig. 7H,I). Deletion of the RING finger domain results in less stabilization of SHCBP1 (Fig. 7H,I). These data suggest that the E3 ubiquitin ligase activity of mLin41 is at least partially required for the regulation of SHCBP1 stability.

The function of SHCBP1 has not been explored, but its expression has been noted in proliferating cells, including cancer cells (Schmandt et al. 1999), and SHCBP1 expression decreases during neural differentiation (Fig. 7J), consistent with the observation that $\mathrm{mLin} 41$ promotes SHCBP1 protein expression and mLin41 expression declines during neural differentiation (Fig. 1A). To test whether SHCBP1 expression is required for FGF signaling in neural progenitor cells, we knocked down the expression of SHCBP1 in NE-4C cells and used p-ERK $1 / 2$ and p-AKT as readouts of FGF signaling. This showed that loss of SHCBP1 expression significantly blunted the response of neural progenitor cells to FGF2 treatment (Fig. $7 \mathrm{~K}, \mathrm{~L}$ ), indicating that SHCBP1 is necessary for FGF signaling in neural progenitor cells. Together, these studies indicate that SHCBP1 is a positive regulator of FGF signaling and that mLin 41 promotes FGF signaling by enhancing SHCBP1 protein stability in neural progenitor cells.

\section{Discussion}

Here we identify mLin 41 as a temporal regulator of neural progenitor cell maintenance during early neural development. Although mLin41 is an E3 ubiquitin ligase that associates with the miRNA effector AGO2, our in vitro and in vivo studies suggest that AGO2 is not the bona fide substrate of mLin 41 and mLin 41 does not regulate the ubiquitination and stability of AGO2 in neural progenitor cells. Our mechanistic studies indicate that mLin41 positively regulates FGF signaling through controlling the stability of SHCBP1, and we show SHCBP1 is necessary for FGF signaling in neural progenitor cells. These findings are particularly important in providing new insights into understanding the temporal regulation of neural progenitor cell maintenance and the spatiotemporal control of FGF signaling during early development.

Loss of mLin41 causes a NTD (Maller Schulman et al. 2008), as also shown here (Fig. 2E,F). However, the underlying basis of the NTD and the mechanisms by which mLin41 regulates neural development had not been previously determined. In this study, we found that mLin 41 controls the balance of proliferation and differentiation of neural progenitor cells and disruption of this balance upon the deletion of mLin41 leads to NTD (Figs. 3, 4). Furthermore, our in vitro and in vivo studies suggest that mLin41 regulates neural development through FGF signaling (Figs. 6, 7). Previous studies in embryonal carcinoma cells reported that mLin41 functions as the E3 ubiquitin ligase to degrade AGO2 (Rybak et al. 2009). However, we did not detect changes in AGO2 protein levels in 293T cells upon mLin4loverexpression in the presence or absence of proteasome inhibitors (Fig. 5B). Moreover, ubiquitination assays failed to show a change in AGO2 ubiquitination in the presence of mLin41 (Fig. 5E,F; Supplemental Fig. 4A,B). Most importantly, in vivo analyses showed that AGO2 protein levels are not significantly changed in the absence of mLin 41 (Fig. 5C,D), nor are the endogenous ubiquitination levels of AGO2 (Fig. $5 \mathrm{G}, \mathrm{H}$; Supplemental Figure 4C). Although it is possible that the regulation of AGO2 by mLin41 is distinct in different cell types, our in vitro and in vivo studies suggest that mLin 41 does not directly regulate AGO2 ubiquitination or stability in neural progenitor cells under physiological conditions. It remains an open question whether mLin41 functions as an E3 ubiquitin ligase to regulate other miRNA pathway proteins in early development. 
A
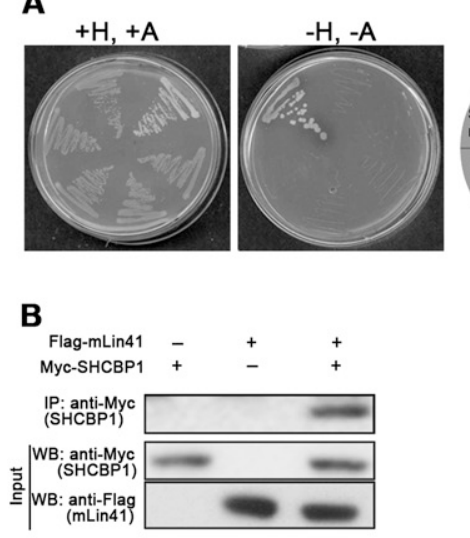
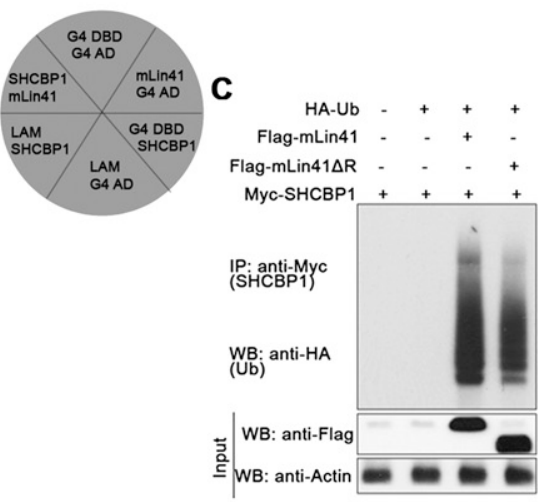

D

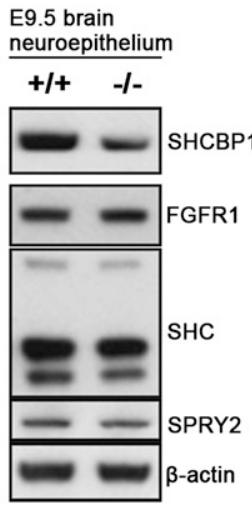

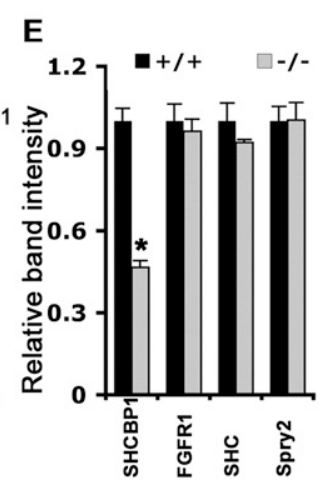

\section{$\mathbf{F}$}

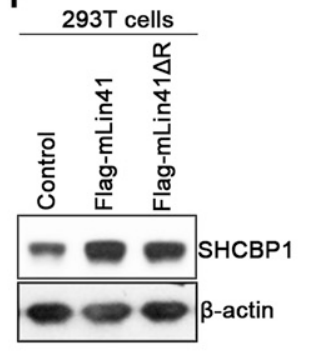

G

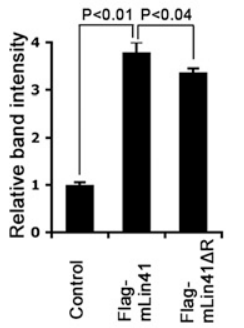

H

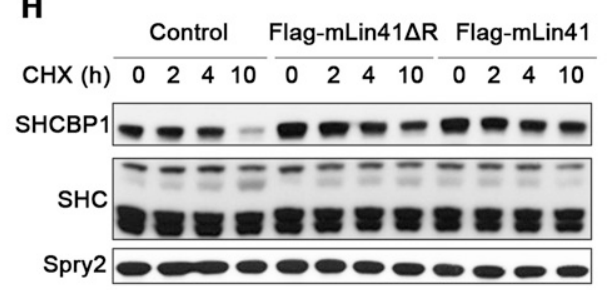

\section{I}

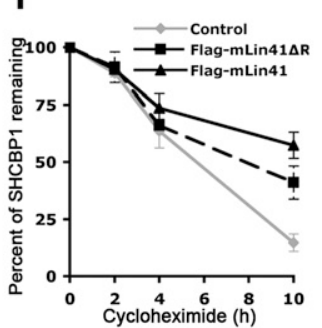

J

Brain tissues
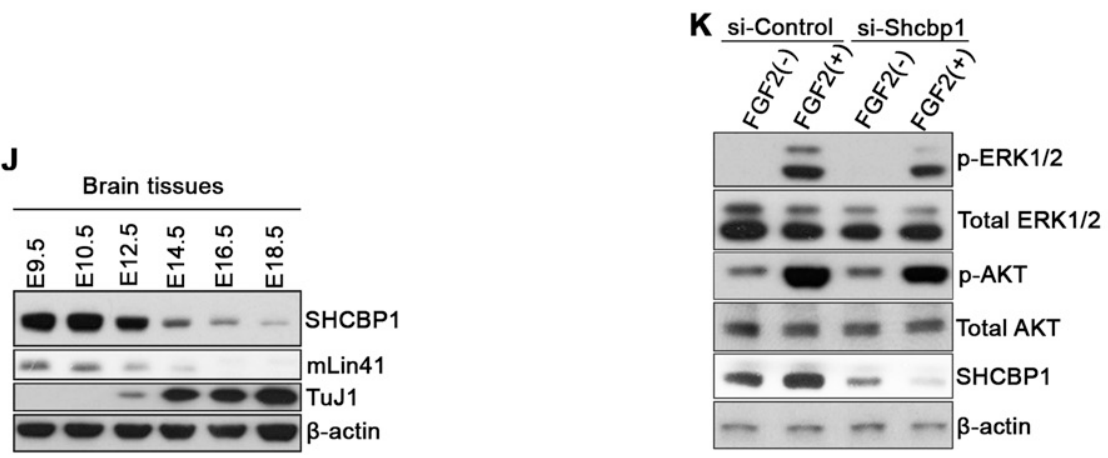

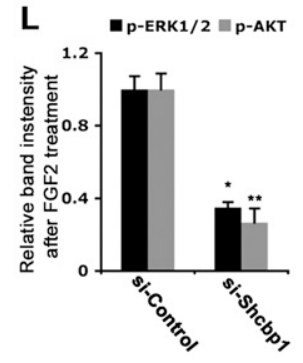

Figure 7. mLin41 enhances the stability of SHCBP1, an important component of FGF signaling in neural progenitor cells. $(A)$ mLin41SHCBP1 interaction in yeast two-hybrid assay. Yeast cells were cotransfected with the indicated plasmids and plated in medium with $(+)$ or without (-) histidine (H) and adenine (A). (AD) Activation domain; (DBD) DNA-binding domain; (G4) Gal4; (LAM) lamin. (B) mLin41 is physically associated with SHCBP1. Protein extracts from HEK293 cells coexpressing Myc-tagged SHCBP1 and Flag-tagged mLin41 were immunoprecipitated using anti-Myc tag beads (IP Myc) followed by immunoblot with anti-Flag antibody (WB mLin41). (C) mLin41 promotes SHCBP1 ubiquitination. Flag-tagged wild-type or RING finger domain-deleted mutant $(\Delta \mathrm{R}) \mathrm{mLin} 41$ were expressed in $293 \mathrm{~T}$ cells along with Myc-SHCBP1 and HA-ubiquitin $(\mathrm{Ub})$ as indicated. The levels of SHCBP1 ubiquitylation were evaluated by immunoprecipitation of SHCBP1 using anti-Myc antibodies followed by anti-HA immunoblotting. (D) Western blot analyses of the expression of FGF signaling components in the cranial neuroepithelium of E9.5 wild-type and mLin41 1acZ/lacZ mutant embryos. $\beta$-Actin serves as a loading control. (E) Quantification of Western blot data from three independent blots in $D_{;}\left({ }^{\star}\right) P<0.005$. $(F)$ Western blot analyses of endogenous expression of SHCBP1 upon the overexpression of mLin41 or RING finger domain-deleted mLin41 $\mathrm{R}$ in the HEK293T cells. (G) Quantification of Western blot data from three independent blots in $F .(H)$ mLin41 enhances the stability of SHCBP1. HEK293T cells transfected with $\mathrm{mLin} 41$ or $\mathrm{mLin} 41 \Delta \mathrm{R}$ for $24 \mathrm{~h}$ were treated with $25 \mathrm{mg} / \mathrm{L} \mathrm{CHX}$, and cells were lysed at different times as indicated. Stability of endogenous of SHCBP1 was determined by Western blot analyses with anti-SHCBP1 antibody. (I) Quantification of Western blot data from three independent experiments in $H$. (J) Western blot analyses of the protein expression of SHCBP1, mLin41, and neural differentiation marker TuJ1 in cranial neuroepithelium at different embryonic stages as indicated. $\beta$-Actin serves as a loading control. (K) Western blot analyses of the expression of p-ERK1/2 and p-AKT in control or Shcbp 1 knockdown NE-4C cells in the presence or absence of FGF2 for $10 \mathrm{~min}$. Total ERK1/2 and total AKT expression serve as controls. Note the induction of p-ERK1/2 and p-AKT expression in response to FGF2 is significantly reduced in the Shcbp1 knockdown cells compared with control cells. $(L)$ Quantification of Western blot data using three independent blots in $K .\left(^{\star}\right) P<0.02 ;\left(^{\star \star}\right) P<0.001$. 
Neural progenitor cells at different stages of development use distinct self-renewal programs to control their proliferation rate, self-renewal potential, and orientation of cell division (Gotz and Huttner 2005; Levi and Morrison 2008). However, the underlying genetic and mechanistic components that control neural progenitor cells at early embryonic stages are ill-defined. In this study, we demonstrate that mLin 41 is temporally expressed in early neural progenitor cells. mLin 41 is required to promote cell proliferation and prevent premature differentiation of neural progenitor cells. The biological significance of mLin41 action is to maintain this unique selfrenewal program in order to expand neural progenitor cell pools necessary for neural tube growth. The absence of mLin41 leads to NTDs in mLin41 $1^{\text {lacZ/lac } Z}$ mutant mice (Fig. 2E,F; Maller Schulman et al. 2008). While reduced proliferation within the neuroepithelium is known to cause NTDs (Copp et al. 2003), it remains largely unknown how neural progenitor cells achieve spatiotemporal control of cell proliferation. Our current results on the temporal control of neural progenitor proliferation by mLin41, together with our previous studies on the spatial control of neural progenitor proliferation (Kim et al. 2007), reveal that neural progenitor cells express distinct genes in a spatiotemporal manner to precisely control cell proliferation and neural tube growth.

Signaling through FGF, WNT, Notch, and SHH pathways is well known to regulate neural progenitor cell proliferation (Doe 2008). However, it has been unclear how neural progenitor cells intrinsically interpret these signaling events in a spatiotemporal manner. We show here that mLin 41 is a positive temporal regulator of FGF signaling within neural progenitor cells. Moreover, we identify SHCBP1 as a protein that interacts with, and is ubiquitinated by, mLin41 (Fig. 7A-C); mLin41 promotes SHCBP1 stability (Fig. 7D-I), and SHCBP1 function is required for FGF signaling in neural progenitor cells (Fig. $7 \mathrm{~K}, \mathrm{~L})$. Overexpression of mLin41 in which the RING finger domain has been deleted still has substantial activity, albeit less than full-length mLin41, suggesting that mLin41 may promote SHCBP1 stability in an E3 ubiquitin ligase activity-independent manner. It is possible that mLin 41 or $\mathrm{mLin} 41 \Delta \mathrm{R}$ could serve as an adaptor protein to recruit unknown E3 ubiquitin ligases to promote SHCBP1 protein stability, which could be studied in the future. As FGF signaling is critical in the regulation of neuroepithelial proliferation, and FGF signaling is disrupted in mLin41 mutants, this can explain the significant decrease in proliferation observed in the mLin41 mutant neural tube. FGF signaling also represses neural differentiation, and signaling must be attenuated for neurogenesis to proceed (Diez del Corral et al. 2002; Kang et al. 2009). Our work suggests that the attenuation of FGF signaling is at least in part due to the temporal decline in expression of mLin41. Together, mLin41 functions as a temporal and positive regulator for neural progenitor maintenance. It does so through enhancing the stability of SHCBP1, an essential component of the FGF signaling, therefore promoting FGF signaling in neural progenitor cells (Fig. 8).

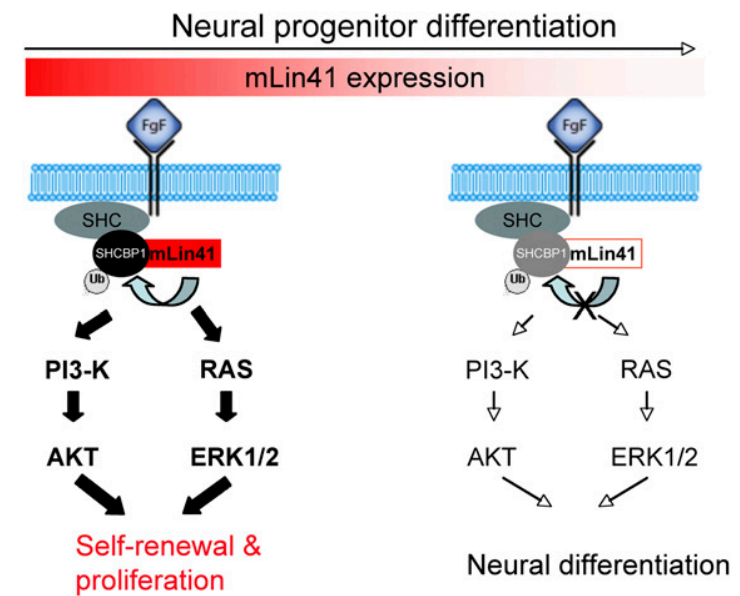

Figure 8. Schematic model of mLin41 functions and its involvement in FGF signaling in neural progenitor cells. mLin41 is highly expressed in neural progenitor cells, in which SHCBP1 is an essential component of FGF signaling. As an E3 ubiquitin ligase, mLin41 binds and ubiquitinates SHCBP1, which leads to its stabilization and the promotion of FGF signaling. mLin41 expression declines during neural differentiation, which results in the reduction of SHCBP1 expression and FGF signaling. Thus, mLin41 promotes neural progenitor cell maintenance through enhancing SHCBP protein stability and FGF signaling.

\section{Materials and methods}

Generation of mLin41 $1^{\text {lacz/lacz }}$ mutant mice

The ES cell line XA144, containing lacZ inserted into the mLin41 locus (Fig. 2A), was obtained from BayGenomics and injected into C57BL/6J blastocysts by the University of North Carolina at Chapel Hill (UNC-CH) Animal Core Facility. The chimeric offspring were mated to $129 \mathrm{~S} 1 / \mathrm{SvIm}$ J mice for germline transmission, and the progeny were screened for heterozygous $m L i n 41^{\text {lac } Z /+}$ mice using genotyping primer set $\mathrm{mLin} 41 \mathrm{~F}, \mathrm{mLin} 41 \mathrm{R} 1$, and $\mathrm{mLin} 41 \mathrm{R} 2$ as described in Supplemental Table 1. Results were obtained from embryos that were third generation in 129S1/SvImJ.

\section{Analysis of mutant phenotype}

Whole-mount and section RNA in situ hybridizations were performed as described (Zohn et al. 2006; Kim et al. 2007). The plasmids for mLin41, Shh, Notch1, Hes-5, Mash1, and Math1 probes are available on request. Histological processing, TUNEL assay, BrdU-labeling experiments, immunofluorescence staining on cell lines, and immunohistochemical labeling of cryosections were performed as described (Chen et al. 2008) using primary antibodies as listed in Supplemental Table 2. Secondary antibodies used were Alexa 488 and Alexa 555 conjugated to specific IgG types (Invitrogen Molecular Probes).

\section{Plasmid constructs}

All plasmids were constructed according to standard molecular methods. Full-length and RING finger domain-deleted mLin41 were cloned into Flag-pCDNA vector using primers described in Supplemental Table 1. The serial deletion constructs of $m L$ in 41 for immunoprecipitation and Shcbp1 were inserted into Myctagged pCMV-3Tag-4 vector (Stratagene) using the primers described in Supplemental Table 1. Flag-tagged Ago2 plasmids were 
generous gifts from the Da-zhi Wang laboratory at the Children's Hospital of Boston at Harvard Medical School.

\section{Yeast two-hybrid screen}

To identify mLin41-interacting proteins, a yeast two-hybrid screen was conducted according to the Matchmaker Gold yeast two-hybrid system user manual (BD Clontech, catalog no. 630489). Specifically, mLin41 full-length fused with GAL4 DNAbinding domain was used as a bait to screen a mouse E11 cDNA library as described by the manufacturer (BD Clontech, catalog no. 630478). The prey plasmids were recovered from those potential interacting clones and then subjected to digestion with Alul or HaeIII to eliminate duplicate clones before final sequencing.

\section{Cell culture and whole-embryo culture}

NE-4C cells were obtained from the American Type Culture Collection (ATCC) and cultured in $\alpha$-MEM (Invitrogen) supplemented with $10 \%$ FBS. To examine the response of neuroepithelium to FGF2, E9.5 wild-type or mLin41 mutant embryos were dissected and cultured for $10 \mathrm{~min}$ with or without $20 \mathrm{ng} / \mathrm{mL}$ FGF2 according the whole-embryo culture method described previously (Pyrgaki et al. 2011). The cranial neuroepithelium were collected for Western blot analyses.

Proteasome inhibition assay, immunoprecipitation, and Western blotting

For proteasome inhibition assay, 293T cells were transfected with plasmids as indicated in Figure 5B. After $24 \mathrm{~h}$ posttransfection, $10 \mu \mathrm{M}$ MG-132 was added to the treatment in the fourth lane (Fig. 5B) for $12 \mathrm{~h}$ before the sample collection. Cells were lysed in lysis buffer $(50 \mathrm{mM}$ Tris- $\mathrm{HCl}$ at $\mathrm{pH} 7.4,150$ $\mathrm{mM} \mathrm{NaCl}, 1 \mathrm{mM}$ EDTA, $1 \%$ Triton X-100, 1 tablet protease inhibitor [Roche] per $10 \mathrm{~mL}$ ). Cell debris was pelleted at 12,500 rpm for $10 \mathrm{~min}$ at $4^{\circ} \mathrm{C}$, and the supernatant was incubated with primary antibodies overnight at $4^{\circ} \mathrm{C}$. The lysates were incubated with Protein A or G Sepharose beads for $2 \mathrm{~h}$, followed by washing of the immunoprecipitates three times with lysis buffer and elution of bound proteins in SDS-PAGE sampling buffer for $10 \mathrm{~min}$ at $100^{\circ} \mathrm{C}$. Western blots were performed as described previously (Chen et al. 2006) using primary antibodies as listed in Supplemental Table 2. Secondary antibodies used were donkey anti-sheep (713-035-147, Jackson ImmunoResearch), goat anti-rabbit (172-1019, Bio-Rad), and goat anti-mouse (1721011, Bio-Rad).

\section{In vitro ubiquitination assay}

mLin41 proteins were purified from HEK293T cells transfected with Flag-tagged mLin41 using Flag-conjugated beads (Sigma). The mLin41 proteins and recombinant AGO2 (50683-M07B, Sino Biological Inc.) were added to $20 \mu \mathrm{L}$ of ubiquitination mixture containing $2 \mu \mathrm{g}$ of ubiquitin (Boston Biochem), $0.5 \mu \mathrm{g}$ of E1 (Boston Biochem), $0.5 \mu \mathrm{g}$ of E2 (Ubch5a), $50 \mathrm{mM}$ Tris (pH 7.5), $1 \mathrm{mM}$ DTT, $5 \mathrm{mM} \mathrm{MgCl} 2,10 \mathrm{mM}$ ATP, $1 \mu \mathrm{M}$ ubiquitin aldehyde (Calbiochem), and 2 mM MG132 (Boston Biochem). The reaction was incubated for $2 \mathrm{~h}$ at $37^{\circ} \mathrm{C}$ and stopped by the addition of SDS sample buffer followed by Western blot analysis with AGO2 antibody.

\section{In vivo ubiquitination assay}

NE-4C cells or $293 \mathrm{~T}$ cells were transfected with various combinations of plasmids as indicated (Fig. 5E; Supplemental Fig. 4B), along with HA-tagged ubiquitin. After $36 \mathrm{~h}$ post-transfection, the whole-cell extracts prepared by lysis buffer were subjected to immunoprecipitation of the substrate protein. The analysis of ubiquitination was carried out by immunoblotting with anti-HA antibodies.

\section{Acknowledgments}

We are very grateful to Bruce Appel and Rytis Prekeris for the advice and discussion on this work. We thank Lori Bulwith and Angela Minic for technical assistance, and our laboratory colleagues for stimulating discussions. This work was supported by the National Institutes of Health (grant no. NS058979). J.C. is a Damon Runyon Cancer Research Fellow, and L.N. is an Investigator of the Howard Hughes Medical Institute.

\section{References}

Ayala R, Shu T, Tsai LH. 2007. Trekking across the brain: The journey of neuronal migration. Cell 128: 29-43.

Bertrand N, Castro DS, Guillemot F. 2002. Proneural genes and the specification of neural cell types. Nature reviews 3: 517-530.

Bielas S, Higginbotham H, Koizumi H, Tanaka T, Gleeson JG. 2004. Cortical neuronal migration mutants suggest separate but intersecting pathways. Annu Rev Cell Dev Biol 20: 593618.

Briscoe J, Pierani A, Jessell TM, Ericson J. 2000. A homeodomain protein code specifies progenitor cell identity and neuronal fate in the ventral neural tube. Cell 101: 435-445.

Chen JF, Mandel EM, Thomson JM, Wu Q, Callis TE, Hammond SM, Conlon FL, Wang DZ. 2006. The role of microRNA-1 and microRNA-133 in skeletal muscle proliferation and differentiation. Nat Genet 38: 228-233.

Chen JF, Murchison EP, Tang R, Callis TE, Tatsuguchi M, Deng Z, Rojas M, Hammond SM, Schneider MD, Selzman CH, et al. 2008. Targeted deletion of Dicer in the heart leads to dilated cardiomyopathy and heart failure. Proc Natl Acad Sci 105: 2111-2116.

Copp AJ, Greene ND, Murdoch JN. 2003. The genetic basis of mammalian neurulation. Nat Rev Genet 4: 784-793.

Deng C, Bedford M, Li C, Xu X, Yang X, Dunmore J, Leder P. 1997. Fibroblast growth factor receptor-1 (FGFR-1) is essential for normal neural tube and limb development. Dev Biol 185: 42-54.

Diez del Corral R, Breitkreuz DN, Storey KG. 2002. Onset of neuronal differentiation is regulated by paraxial mesoderm and requires attenuation of FGF signalling. Development 129: $1681-1691$.

Doe CQ. 2008. Neural stem cells: Balancing self-renewal with differentiation. Development 135: 1575-1587.

Gotz M, Huttner WB. 2005. The cell biology of neurogenesis. Nat Rev Mol Cell Biol 6: 777-788.

Guillemot F. 2005. Cellular and molecular control of neurogenesis in the mammalian telencephalon. Curr Opin Cell Biol 17: 639-647.

Jessell TM. 2000. Neuronal specification in the spinal cord: Inductive signals and transcriptional codes. Nat Rev Genet 1: 20-29.

Jessell TM, Dodd J. 1990. Floor plate-derived signals and the control of neural cell pattern in vertebrates. Harvey Lect 86: $87-128$.

Kanamoto T, Terada K, Yoshikawa H, Furukawa T. 2006. Cloning and regulation of the vertebrate homologue of lin41 that functions as a heterochronic gene in Caenorhabditis elegans. Dev Dyn 235: 1142-1149. 
Kang W, Wong LC, Shi SH, Hebert JM. 2009. The transition from radial glial to intermediate progenitor cell is inhibited by FGF signaling during corticogenesis. I Neurosci 29: 14571-14580.

Kim TH, Goodman J, Anderson KV, Niswander L. 2007. Phactr4 regulates neural tube and optic fissure closure by controlling PP1-, Rb-, and E2F1-regulated cell-cycle progression. Dev Cell 13: 87-102.

Kirkin V, Dikic I. 2007. Role of ubiquitin- and Ubl-binding proteins in cell signaling. Curr Opin Cell Biol 19: 199-205.

Kuan CY, Roth KA, Flavell RA, Rakic P. 2000. Mechanisms of programmed cell death in the developing brain. Trends Neurosci 23: 291-297.

Levi BP, Morrison SJ. 2008. Stem cells use distinct self-renewal programs at different ages. Cold Spring Harb Symp Quant Biol 73: 539-553.

Maller Schulman BR, Liang X, Stahlhut C, DelConte C, Stefani G, Slack FJ. 2008. The let-7 microRNA target gene, Mlin41/ Trim71 is required for mouse embryonic survival and neural tube closure. Cell Cycle 7: 3935-3942.

Mason I. 2007. Initiation to end point: The multiple roles of fibroblast growth factors in neural development. Nat Rev Neurosci 8: 583-596.

Novitch BG, Chen AI, Jessell TM. 2001. Coordinate regulation of motor neuron subtype identity and pan-neuronal properties by the bHLH repressor Olig2. Neuron 31: 773-789.

Ohnuma S, Harris WA. 2003. Neurogenesis and the cell cycle. Neuron 40: 199-208.

Ohtsuka T, Ishibashi M, Gradwohl G, Nakanishi S, Guillemot F, Kageyama R. 1999. Hes1 and Hes5 as notch effectors in mammalian neuronal differentiation. EMBO J 18: 2196-2207.

Pfaff SL, Mendelsohn M, Stewart CL, Edlund T, Jessell TM. 1996. Requirement for LIM homeobox gene Isll in motor neuron generation reveals a motor neuron-dependent step in interneuron differentiation. Cell 84: 309-320.

Pyrgaki C, Trainor P, Hadjantonakis AK, Niswander L. 2011. Dynamic imaging of mammalian neural tube closure. Dev Biol 344: 941-947.

Rybak A, Fuchs H, Hadian K, Smirnova L, Wulczyn EA, Michel G, Nitsch R, Krappmann D, Wulczyn FG. 2009. The let-7 target gene mouse lin- 41 is a stem cell specific E3 ubiquitin ligase for the miRNA pathway protein Ago2. Nat Cell Biol 11: 1411-1420.

Schmandt R, Liu SK, McGlade CJ. 1999. Cloning and characterization of $\mathrm{mPAL}$, a novel Shc $\mathrm{SH} 2$ domain-binding protein expressed in proliferating cells. Oncogene 18: 1867-1879.

Schulman BR, Esquela-Kerscher A, Slack FJ. 2005. Reciprocal expression of lin-41 and the microRNAs let-7 and mir-125 during mouse embryogenesis. Dev Dyn 234: 1046-1054.

Slack FJ, Basson M, Liu Z, Ambros V, Horvitz HR, Ruvkun G. 2000. The lin-41 RBCC gene acts in the C. elegans heterochronic pathway between the let-7 regulatory RNA and the LIN-29 transcription factor. Mol Cell 5: 659-669.

Sorkin A, von Zastrow M. 2009. Endocytosis and signalling: Intertwining molecular networks. Nat Rev Mol Cell Biol 10: 609-622.

Varga BV, Hadinger N, Gocza E, Dulberg V, Demeter K, Madarasz E, Herberth B. 2008. Generation of diverse neuronal subtypes in cloned populations of stem-like cells. $B M C$ Dev Biol 8: 89. doi: 10.1186/1471-213X-8-89.

Yoon K, Gaiano N. 2005. Notch signaling in the mammalian central nervous system: Insights from mouse mutants. Nat Neurosci 8: 709-715.

Zohn IE, Li Y, Skolnik EY, Anderson KV, Han J, Niswander L. 2006. p38 and a p38-interacting protein are critical for downregulation of E-cadherin during mouse gastrulation. Cell 125: 957-969. 


\section{Erratum}

Genes \& Development 26: 803-815 (2012)

The ubiquitin ligase mLin41 temporally promotes neural progenitor cell maintenance through FGF signaling Jianfu Chen, Fan Lai, and Lee Niswander

The authors have identified an error in Supplemental Figure 1B of the above-mentioned article. Two identical images were inadvertently shown in the left-most panels. This has been corrected by changing the one incorrect panel (the correct image is now online). This correction does not change the findings or conclusions of this study. The authors apologize for this error. 


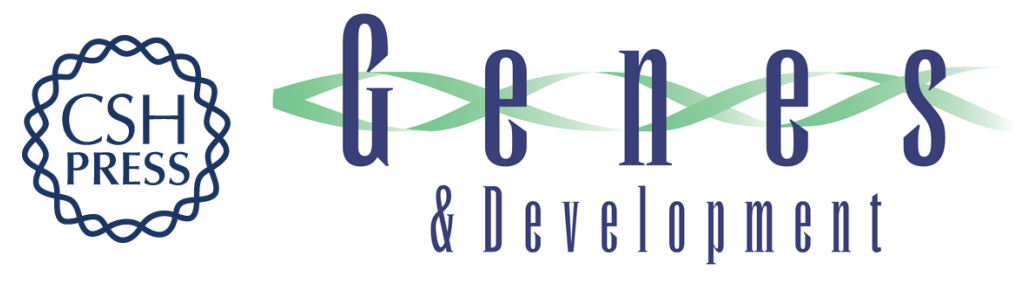

\section{The ubiquitin ligase mLin41 temporally promotes neural progenitor cell maintenance through FGF signaling}

Jianfu Chen, Fan Lai and Lee Niswander

Genes Dev. 2012, 26:

Access the most recent version at doi:10.1101/gad.187641.112

Supplemental http://genesdev.cshlp.org/content/suppl/2012/04/13/26.8.803.DC1
Material

Related Content The ubiquitin ligase mLin41 temporally promotes neural progenitor cell maintenance through FGF signaling

Jianfu Chen, Fan Lai and Lee Niswander

Genes Dev. June , 2012 26: 1392

References This article cites 35 articles, 6 of which can be accessed free at: http://genesdev.cshlp.org/content/26/8/803.full.html\#ref-list-1

Articles cited in:

http://genesdev.cshlp.org/content/26/8/803.full.html\#related-urls

License

Email Alerting Receive free email alerts when new articles cite this article - sign up in the box at the top

Service right corner of the article or click here.

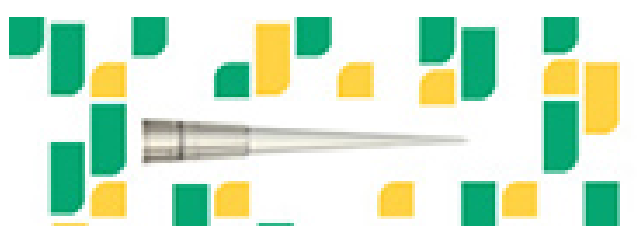

Focused on your science. 